\title{
Transtornos alimentares e as contribuições da Terapia Cognitivo- Comportamental para o tratamento
}

\author{
Eating disorders and the contributions of Cognitive Behavior Therapy to treatment
}

Juliene Avila Pires ${ }^{\dagger *}$, Tamires Jordão Laport

Como citar esse artigo. Pires, J.A.;

Laport, T.J. Transtornos alimentares e as contribuições da Terapia CognitivoComportamental para o tratamento. Revista Mosaico 2019 Jul/Dez.; 10 (2): SUPLEMENTO 116-123

\begin{abstract}
Resumo
A Terapia Cognitivo-Comportamental (TCC) tem sido amplamente utilizada como tratamento para os transtornos alimentares, principalmente para a anorexia nervosa (AN) e a bulimia nervosa (BN). O presente artigo tem por objetivo discutir sobre os transtornos alimentares sob a ótica do tratamento cognitivo-comportamental.Para uma incursão a estes campos de investigação, a metodologia desse estudo foi uma revisão narrativa da literatura. A TCC tem se mostrado eficaz tanto para o tratamento da AN quanto da $\mathrm{BN}$, principalmente em relaçãoà modificação do sistema de crenças e dos pensamentos associados à alimentação, à imagem corporal, na melhoria da autoestima e no desenvolvimento das habilidades sociais, além de promover o aumento da adesão ao tratamento nutricional e clínico. Tendo em vista que a utilização da TCC como tratamento para os transtornos alimentares ainda érelativamente recente no Brasil se faz necessário mais estudos sobre a temática.

Palavras-chave: transtornos alimentares;anorexia nervosa; bulimia nervosa; terapia cognitivo-comportamental.
\end{abstract}

\begin{abstract}
Cognitive Behavioral Therapy (CBT) has been widely used as a treatment for eating disorders, especially for anorexia nervosa (AN) and bulimia nervosa (BN). This article aims to discuss about eating disorders from the perspective of cognitive behavioral treatment. For an incursion into these fields of investigation, the methodology of this study was a narrative review of the literature. CBT has been shown to be effective for the treatment of $\mathrm{AN}$ and $\mathrm{BN}$, especially in relation to the modification of the belief system and thoughts associated with eating, body image, improvement of self-esteem and the development of social skills, increased adherence to nutritional and clinical treatment. Given that the use of CBT as a treatment for eating disorders is still relatively recent in Brazil, further studies on the subject are needed.

Keywords: eating disorders; anorexia nervosa; bulimia nervosa; cognitive behavioral therapy.
\end{abstract}

\section{Introdução}

Os transtornos alimentares (TA) são síndromes psiquiátricas referentes ao comportamento alimentar que comprometem significativamente a integridade física, psicológica e social. Sua etiologia é multifatorial, com bases genética, psicológica e sociocultural. Devido a essa etiologia o tratamento necessita de intervenções multiprofissionais, é ideal que essa equipe seja composta por educadores físicos, enfermeiros, dentistas, médicos clínicos, nutricionistas, psicólogos, psiquiatras, entre outros (OLIVEIRA; DEIRO, 2013).

A anorexia nervosa e a bulimia nervosa são os transtornos alimentares mais frequentes, com incidência de $5 \%$ a $10 \%$ e prevalência em mulheres jovens, com idades entre 12 e 28 anos (MELIN; ARAÚJO, 2002;
PEDRINOLA, 2012; OLIVEIRA; DEIRO, 2013). A prevalência desses transtornos tem crescido de forma significativa, praticamente dobrou nessas últimas duas décadas (MORGAN; VECCHIATTI; NEGRÃO, 2002). Pode-se falar em epidemia dos TA em uma sociedade contemporânea com a exacerbação da "cultura da magreza", que associa a magreza ao sucesso. É de suma importância, portanto, à elaboração de estudos sobre o tema, visto a grande influência que fatores culturais, sociais, econômicos e políticos exercem sobre os corpos (IDA; SILVA, 2007).

Para uma incursão a estes campos de investigação, a metodologia desse estudo foi uma revisão narrativa da literatura baseada em artigos científicos, teses, dissertações e livros. Os dados foram pesquisados nas bases de dados, SciElo, PsycINFO, BVS-Psi e

Afiliação dos autores: $\uparrow$ Graduanda do Curso de Psicologia, Universidade de Vassouras, Vassouras, RJ, Brasil

* Docente do Curso de Psicologia, Universidade de Vassouras, Vassouas,RJ, Brasil

* Email de correspondencia: julieneapires@hotmail.com 
LILACS e os descritores utilizados para a busca foram: Transtornos Alimentares, Anorexia Nervosa, Bulimia Nervosa e Terapia Cognitivo-Comportamental.

No decorrer desse artigo serão abordados os sintomas, curso e tratamento dos transtornos alimentares. Sendo dada ênfase as contribuições da Terapia Cognitivo-Comportamental no que tange aos aspectos psicológicos do tratamento para a anorexia nervosa e a bulimia nervosa, visto que são os transtornos alimentares de maior incidência.Desse modo, o presente artigo à luz da literatura científica atual tem por objetivo discutir sobre os transtornos alimentares sob a ótica do tratamento cognitivo-comportamental.

\section{Transtornos alimentares: anorexia nervosa e bulimia nervosa}

Segundo o DSM-V (2014) são tidos como transtornos alimentares: pica, transtorno de ruminação, transtorno alimentar restritivo/evitativo, anorexia nervosa, bulimia nervosa e transtorno de compulsão alimentar. Nesse artigo enfatizaremos a anorexia nervosa e a bulimia nervosa.

Os transtornos alimentares são caracterizados por uma perturbação recorrente na alimentação ou no comportamento alimentar, que tem como consequência alterações no consumo ou na absorção alterada de alimentos, gerando um comprometimento à saúde física e psicossocial do indivíduo (DSM-V, 2014). São considerados síndromes psiquiátricas referentes ao comportamento humano, indivíduos com esse diagnóstico apresentam sintomas comportamentais como: alterações quantitativas da ingesta alimentar, comportamentos purgativos, vômitos autoinduzidos, atividade física excessiva, comportamentos considerados de risco (FORTES et al., 2016).

Os transtornos alimentares possuem etiologia multifatorial, ou seja, sua causa é determinada pela interação entre fatores biológicos, psicológicos, familiares e socioculturais que produzem e, muitas vezes, perpetuam o transtorno. Esses fatores podem se distinguir entre: fatores predisponentes - são os que aumentam a chance do aparecimento do transtorno, mas não o tornam inevitável, podemos destacar a história de transtorno alimentar e/ou transtorno do humor na família e o contexto sociocultural; os fatores precipitantes - são os que marcam o aparecimento dos sintomas dos transtornos alimentares, como por exemplo, as dietas e eventos estressores e por último, os fatores mantenedores - são os que determinam a perpetuação ou não do transtorno, vale destacar como exemplos, as dietas restritas, os aspectos culturais e a magreza vista como um símbolo de sucesso (FLEITLICH et al., 2000;MORGAN; VECCHIATTI; NEGRÃO, 2002; SILVA et al., 2007).
Estudos mostram um considerável aumento na incidência dos transtornos alimentares, sendo possível apontar como um dos transtornos que afetam predominantemente mulheres jovens e com altos índices de morbidade e mortalidade (HOEK; VAN HOEKEN, 2003). Com um acometimento de nove mulheres para cada homem (BUCARETCHI; CORDÁS, 2003). Dentre os transtornos alimentares, os mais comuns são anorexia nervosa e bulimia nervosa, com índices de prevalência para a anorexia nervosa estão entre $0,5 \%$ e $3,7 \%$ e para a bulimia nervosa entre $1,1 \%$ e $4,2 \%$ (PINZON; NOGUEIRA, 2004).

A anorexia nervosa (AN) é caracterizada principalmente por uma recusa alimentar que leva à caquexia- perda de peso e atrofia muscular (CLAUDINO; BORGES, 2002). Segundo o Manual Diagnóstico e Estatístico de Transtornos Mentais (2014) são tidos como critérios diagnósticos para a anorexia nervosa: a restrição da ingesta calórica de modo persistente; medo de ganhar peso ou de engordar ou comportamento que corrobore para a perda de peso; e distúrbio na percepção do peso corporal ou da própria forma.

De acordo com o DSM-V (2014) a anorexia nervosa pode apresentar dois subtipos: o do tipo restritivo que é caracterizado pela restrição alimentar e pela ausência de episódios de comer compulsivo ou práticas purgativas, neste caso, o indivíduo perde peso por meio de dietas, jejuns e/ou exercícios excessivos; e o do tipo purgativo que é caracterizado por episódios de restrição alimentar seguidos por episódios de comer compulsivos e comportamentos purgativos, ou seja, vômitos autoinduzidos ou uso indevido de laxantes, diuréticos ou enemas. É comum a alternância entre os dois subtipos e essa classificação serve como indicativo para sintomas atuais e não para definir o curso longitudinal do transtorno.

A característica de semi-inanição da anorexia nervosa e os comportamentos purgativos às vezes associados a ela podem provocar perturbações fisiológicas, tais como desnutrição, desidratação, alteraçõeseletrolíticas, infertilidade, hipotermia, laguno, complicações cardiovasculares, e disfunções endócrinas, principalmente no eixo hipotalâmico-hipofisáriogonodal, comamenorreianas mulheres e perda do interesse e potência sexual nos homens(CLAUDINO; BORGES, 2002; VILELA et al., 2004). Além das complicações clínicas,há um déficit das funções cognitivas, principalmente, na atenção, na memória, na viso-construção e na aprendizagem (SILVA et al., 2007).

É comum a associação de outros transtornos psiquiátricos ao transtorno alimentar, como transtornos afetivos, transtornos de ansiedade e/ou transtorno de personalidade, mesclando os seus sintomas aos dos transtornos associados (APPOLINÁRIO; CLAUDINO, 2000).A depressão maior representa o transtorno afetivo 
mais comum na anorexia nervosa, e pode surgir desde o início do transtorno alimentar, principalmente o de tipo restritivo. Dentre os transtornos de ansiedade, a fobia social e o transtorno obsessivo compulsivo (TOC) foram os de maior relevância. Os transtornos de personalidade do tipo evitador e compulsivo são os mais recorrentes entre indivíduos com anorexia nervosa. $\mathrm{E}$ os traços de personalidade encontrados com maior frequência são os de obsessividade, dependência, rigidez, controle sobre impulsos e perfeccionismo (FLEITLICH et al., 2000). Devido a esses transtornos comórbidosé de extrema importância o diagnóstico diferencial tanto com doenças fisiológicas como psiquiátricas, para que se possibilite um diagnóstico preciso e um prognóstico coerente ao transtorno apresentado (DSM-V, 2014).

A bulimia nervosa (BN) é caracterizada principalmente pela hiperfagia em um período delimitado de tempo, tal comportamento não é dado por uma fome fisiológica, mas por uma série de estados emocionais ou situações estressantes (CLAUDINO; BORGES, 2002). Esses episódios de hiperfagia são nomeados pela literatura inglesa como Binge Eating são traduzidos para o português como compulsão alimentar periódica (GONÇALVES, 2012).Segundo o Manual Diagnóstico e Estatístico de Transtornos Mentais (2014) são tidos como critérios diagnósticos para a bulimia nervosa as recorrências de compulsão alimentar, os comportamentos compensatórios que impeçam o ganho de peso, e a autoavaliação pela forma e pelo peso corporais, visto que a compulsão alimentar periódica e os comportamentos compensatórios devem ocorrer, em média, uma vez por semana por três meses.

Os episódios de compulsão alimentar são marcantes da bulimia nervosa e são definidos como uma ingesta excessiva de alimentos se comparada ao que a pessoa comeria em situações normais (APPOLINÁRIO; CLAUDINO, 2000). Esses episódios são acompanhados de uma sensação de perda de controle sobre a quantidade de alimentos ingeridos (SILVA et al., 2007). E geralmente, um episódio de compulsão alimentar é seguido por comportamentos compensatórios/ purgatórios que são em geral, vômitos autoinduzidos; uso indevido de laxantes, diuréticos ou enemas ou uso de outras medicações; dietas restritas; jejuns prolongados ou excessiva prática de exercício físico na tentativa de impedir o ganho de peso e compensar o comportamento compulsório(CID-11, 2018). Gerando assim, um ciclo compulsão alimentar- purgação e como consequência a isso, sentimentos como frustração, tristeza, ansiedade, tédio e solidão (APPOLINÁRIO; CLAUDINO, 2000).

Segundo Nunes et al. (2008), os episódios de compulsão alimentar apresentam os tipos, objetivos esubjetivos. Os episódios de bingeeating objetivos são caracterizados pela ingesta alimentar excessiva para aquele momento ou circunstância, levando em conta a refeição que antecedeu o episódio e a sensação da perda de controle sobre o que e quanto se está comendo. No entanto, alguns indivíduos com bulimia nervosa podem apresentar episódios de bingeeating subjetivos que são caracterizados pela ingestão de "alimentos proibidos", ainda que em quantidade normal, é caracterizado por compulsão alimentar por valorizarem a perda de controle no momento, o sentimento de inevitabilidade frente à compulsão e o fato de terem ingerido um alimento que pode favorecer o ganho de peso. Embora sejam apenas usados como critério diagnóstico para bulimia nervosa os episódios objetivos (os que apresentam perda de controle e excesso alimentar) os autores ressaltam a importância do aspecto qualitativo para os indivíduos com o transtorno.

Os episódios de compulsão alimentar e os comportamentos compensatórios podem desencadear sintomas como a irregularidade menstrual ou amenorreia; deficiência nutricional;distúrbios hidroeletrolíticos; lacerações esofágicas; ruptura gástrica; arritmias cardíacas; miopatias esqueléticas e cardíacas; sintomas gastrointestinais; dentre outros(DSM-V, 2014). Além das manifestações fisiológicas há também um comprometimento das funções cognitivas como atenção e as funções executivas (SILVA et al., 2007).

É comum a associação de transtornos afetivos, como a depressão, essas perturbações do humor podem acometer concomitantemente ou em seguida ao desenvolvimento da bulimia nervosa. Além desses sintomas, também podem apresentar sintomas de ansiedade ou transtornos de ansiedade. É relevante o número de indivíduos que apresentam aspectos da personalidade que contemplam os critérios de um ou mais transtornos alimentares, sendo o transtorno de personalidade borderline o mais frequente (DSM-V, 2014).

Devido às características da bulimia nervosa se assemelhar a outros transtornos psiquiátricos é importante o diagnóstico diferencial para um diagnóstico efetivo que corrobore para um coerente tratamento (DSM-V, 2014). Como por exemplo, o diagnóstico diferencial entre bulimia nervosa e anorexia nervosa do tipo purgativo, visto que, o que as diferenciam é que os indivíduos com bulimia nervosa mantêm um peso corporal igual ou acima da faixa mínima normal, não atendendo aos critérios diagnósticos para anorexia nervosa (CID-11, 2018).

O diagnóstico para os transtornos alimentares é muito complexo, devido principalmente a sua etiologia multifatorial, deve ser feito por meio de entrevistas, como por exemplo, a entrevista Eating Disorder ExaminationEDE que é considerada o modelo-padrão, também tem a Entrevista clínica Estruturada para Transtornos do DSM-IV e, para crianças e adolescentes, a Sessão de Transtornos Alimentares do DAWBA. Os instrumentos autoaplicáveis podem servir para auxiliar o diagnóstico, mas não são instrumentos diagnósticos (RANGÉ et 
al., 2011). Na anorexia nervosa o diagnóstico deve compreender em uma avaliação criteriosa sobre a ingesta alimentar (quantitativa e qualitativa), a prática de atividade física, a concepção de imagem corporal e do peso, e a sociabilidade do paciente (SILVA et al., 2007). O diagnóstico para indivíduos com bulimia nervosa baseia-se na Classificação Internacional das Doenças (CID-10) e no Manual Diagnóstico e Estatístico de Transtornos Mentais (DSM-V) com o objetivo de detectar os sintomas específicos do transtorno (SILVA et al., 2007).

O tratamento para os transtornos alimentares necessita de intervenções que analisem os aspectos clínicos, nutricionais, psiquiátricos e psicológicos de cada indivíduo para que a partir disso se encontre um tratamento mais adequado. Com estabelecimento de algumas etapas para o tratamento: a estabilização do estado clínico e nutricional, o restabelecimento dos padrões de peso e alimentares, a identificação e resolução dos fatores psicossociais mantenedores, a melhora das condições psiquiátricas associadas, a correção dos pensamentos disfuncionais e prevenção de recaídas. Além disso, é importante incluir a família nesse processo para uma colaboração e participação ativa com finalidade de que os pais/cônjuges tornem o meio facilitador de mudanças. (NICE, 2004; OLIVEIRA; DEIRO, 2013).

O tratamento para anorexia nervosa pode apresentar características para internação hospitalar integral ou parcial, quando o indivíduo se encontra com peso corporal abaixo de $75 \%$ do mínimo ideal, ou quando está perdendo peso rapidamente, ou quando necessita de monitoramento das condições clínicas. Outra possibilidade é o tratamento ambulatorial que é indicado para pacientes com bom suporte social, que não estão perdendo peso rapidamente e não preenchem os critérios para internação descritos acima (APPOLINÁRIO; CLAUDINO, 2000; RANGÉ et al., 2011).

Devido o caráter etiológico multifatorial da anorexia nervosa pressupõe-se que as abordagens terapêuticas para o tratamento sejam multidisciplinares, com ênfase nos aspectos nutricionais,objetivando a recuperação o peso corporal por meio da redução da restrição alimentar e da frequência de atividade física; aspectos farmacológicos, com o uso de antidepressivos ou ansiolíticos usados na presença de comorbidades psiquiátricas associados, pois nenhum psicofármaco se mostrou eficaz aos sintomas exclusivos da $\mathrm{AN}$; e aspectos psicológicos, principalmente as técnicas cognitivo-comportamentais no que tange o distúrbio da imagem corporal, a modificação do sistema de crenças, abordagens da autoestima e técnicas para prevenção de recaídas (FLEITLICH et al., 2000; DUCHESNE; ALMEIDA, 2002; SALZANO; CORDÁS, 2004; SILVA et al., 2007).
No tratamento para bulimia nervosa a necessidade de internação hospitalar depende da presença de complicações médico-psiquiátricas, de ciclos incoercíveis de compulsão alimentar-purgação, de abusos de laxativos e/ ou outras drogas e outros comportamentos de risco. Sendo que na maioria dos casos o tratamento é extra-hospitalar (APPOLINÁRIO; CLAUDINO, 2000).

$\mathrm{O}$ tratamento da $\mathrm{BN}$ é caracterizado pela associação de intervenções nutricionais, da terapia medicamentosa adequada com a utilização de agentes antidepressivos como os tricíclicos (TCAs) e os inibidores seletivos de receptação de serotonina (ISRS) que possibilitam a redução da frequência dos sintomas comportamentais (episódios de compulsão e comportamentos purgativos), e atuam nos sintomas da ansiedade e depressão, quando presentes e a associação com a terapia cognitivo-comportamental que lança mão de estratégias para controle de episódios de compulsão alimentar e dos comportamentos compensatórios, aborda também a autoestima, a modificação da relação com a imagem corporal e a modificação do sistema de crenças disfuncionais (APPOLINÁRIO; CLAUDINO, 2000; DUCHESNE; ALMEIDA, 2002; SALZANO; CORDÁS, 2004; SILVA et al., 2007).

\section{TCC para o tratamento dos Transtornos Alimentares}

A Terapia Cognitivo-Comportamental (TCC) é uma intervenção psicoterápica que aborda fatores cognitivos, emocionais e comportamentais no tratamento dos transtornos psiquiátricos. Com intervenção semiestruturada eobjetiva, busca identificar e corrigir os fatores que favorecem o desenvolvimento e manutenção das cognições e comportamentos dos transtornos alimentares (DUCHESNE; ALMEIDA, 2002; OLIVEIRA; DEIRO, 2013). Que se baseia em dois princípios centrais: que nossas cognições influenciam e controlam nossas emoções e comportamentos; e que os nossos comportamentos podem afetar nossos pensamentos e emoções (WRIGHT; BASCO; THASE, 2008)

Aaron T. Beck foi o pioneiro nos estudos que aplicavam as intervenções cognitivas e comportamentais aos transtornos. No início de 1960, fez suas primeiras formulações, com a conceitualização cognitiva da depressão em que os sintomas estavam ligados a uma tríade cognitiva negativa e se relacionavam a três estilos de pensamentos negativos, a cerca de si mesmo, do mundo e do futuro. Beck amplia essa proposta terapêutica com o objetivo de reverter cognições e comportamentos disfuncionais a outros transtornos, como os transtornos de ansiedade, os transtornos alimentares, a esquizofrenia, o transtorno bipolar, a dor 
crônica, os transtornos de personalidade e o abuso de substâncias, além da depressão primeiramente estudada (WRIGHT; BASCO; THASE, 2008; BECK, 2014).

$\mathrm{O}$ modelo cognitivo-comportamental utilizado pela TCC demonstra como os processos cognitivos acontecem.Esse modelo básico é usado na conceitualização dos problemas clínicos e na implementação de técnicas específicas, sendo possível pontuar os acontecimentos internos ou eventos em análise que provocamuma avaliação cognitiva, que são os pensamentos automáticos que ocorrem de forma rápida e espontâneafrente ao evento em questão, gerandoemoções e respostas psicológicas como consequência, e as respostas comportamentais, que podem reforçar tais pensamentos(WRIGHT; BASCO; THASE, 2008).

Beck et al (1979) analisaram que existem alguns equívocos nos pensamentos automáticos $\mathrm{e}$ outras cognições. E classificaram em seis categorias principais de erros cognitivos são a abstração seletiva, inferência arbitrária, supergeneralização, maximização e minimização, personalização e pensamentos dicotômicos.

$\mathrm{Na}$ Terapia Cognitivo-Comportamental, os esquemas/crenças são definidos como matrizes para os pensamentos automáticos, que são caracterizados como regras fundamentais que começam a ser construídas na infância e são influenciadas por experiências de vida, ensinamentos, atividades educativas, traumas e sucessos. E são divididos em três grupos principais: os esquemas simples que são regras sobre a natureza física do ambiente, atividades cotidianas ou leis da natureza e que possuem pouco ou nenhum efeito sobre a psicopatologia; crenças e pressupostos intermediários são regras condicionadas que influenciam a autoestima e regulação emocional; crenças nucleares sobre si mesmo são regras globais, rígidas e absolutas sobre os eventos e que interferem na autoestima. Ao passo em que os pensamentos automáticos possuem erros cognitivos também é característico dos esquemas a sua disfuncionalidade em alguns transtornos (WRIGHT; BASCO; THASE, 2008).

As técnicas cognitivas e comportamentais têm sido expressivamente avaliadas e reconhecidas como eficazes no tratamento para os transtornos alimentares (DUCHESNE; ALMEIDA, 2002).Segundo Judith S. Beck (2014), todos os transtornos psicológicos têm em comum a disfuncionalidade dos pensamentos, que influenciam no humor, nos pensamentos automáticos e nos comportamentos do indivíduo.

O tratamento dos transtornos alimentares com a terapia cognitivo-comportamental se estabelece pelo tratamento transdiagnóstico que consiste na abordagem terapêutica orientada através da conceitualização de cada indivíduo, com a análise de fatores precipitantes e mantenedores do transtorno alimentar e os sintomas associados, ou seja, o tratamento é direcionado a partir dos sintomas psicopatológicosdo paciente (RANGÉ et al., 2011). Esse modelo de tratamento pode ser divido em 4 estágios:

-Estágio1: Nesse primeiro momento são implementadas técnicas que buscam facilitar a adesão ao tratamento, normalizar os padrões alimentares e eliminar os métodos compensatórios.Com duas sessões por semana, esse estágio é mais intenso (RANGÉ et al., 2011).

Inicialmente busca-se informar o paciente acerca dos efeitos psicológicos, físicos e sociais de manter os sintomas do transtorno. Evidenciando os aspectos positivos na mudança dos comportamentos atuais e enfatizando a piora da qualidade de vida após o desenvolvimento do TA. Além de informar quea indução de vômito não elimina todo o alimento ingerido, e o uso de laxantes causa perda temporária de líquidos, sendo assim, evidenciando a ineficácia no que diz respeito à perda de peso corporal (RANGÉ et al., 2011).

A abordagem do terapeuta deve ser o "empirismo colaborativo" que consiste na elaboração de estratégias de modo colaborativo e ativo junto ao paciente, visto que o motivo que mais gera abando ou não cooperação no tratamento é a insatisfação com o vínculo (RANGÉ et al, 2011; WILLHELM; FORTES; PERGHER, 2015).

O terapeuta deve ajudar o paciente a identificar seus pensamentos e evidenciar a disfuncionalidade dos mesmos. Os pensamentos dicotômicos ("tudo ou nada") são frequentes em pacientes com TA. De modo geral são autoimpostas dietas rígidas e inconsistentes, e se ocorre um pequeno lapso na dieta, o indivíduo interpreta como sendo uma deficiência pessoal e aumenta ainda mais a rigidez dietética, reforçando, então, a baixa autoestima (RANGÉ et al., 2011; OLIVEIRA; DEIRO, 2013).

Pode ser utilizada a técnica de automonitoração (ou diário alimentar) que consiste em registrar todos os alimentos e líquidos ingeridos no dia, e tem por objetivo desenvolver novos hábitos alimentares, além de incluir nesse registro os eventos desencadeadores, pensamentos e sentimentos associados. As dietas restritas são uns dos fatores mantenedores e para reverter esse ciclo é importante o desenvolvimento de um padrão alimentar regular com a orientação nutricional, essa mudança só que torna possível quando há modificação de vários tipos de pensamentos disfuncionais (DUCHESNE; ALMEIDA, 2002; RANGÉ et al., 2011).

O monitoramento semanal do peso também pode ser uma técnica usada para ajudar o paciente a avaliar a quantidade de alimento que pode ser ingerido sem ganho de peso. Paralelo a essa técnica é relevante trabalhar a imagem corporal do paciente. Para pacientes que apresentam episódios de compulsão alimentar as técnicas de controle de estímulos são importantes, pois diminui a exposição do paciente a situações que ocorreriam o descontrole alimentar (RANGÉ et al., 
2011).

-Estágio 2: Nesse estágio serão avaliadas os resultados obtidos a partir das técnicas implementadas no estágio 1. Com uma análise, revisão e seleção de técnicas adicionais (RANGÉ et al., 2011).

-Estágio 3: Nessa etapa as técnicas anteriormente aplicadas devem continuar sendo praticadas, o tratamento é cumulativo. O estágio 3 é direcionado a reestruturação do sistema de crençasassociadas ao TA. É fundamental que o paciente entenda a relação entre seus pensamentos, sentimentos e disfunções de comportamentos para que ocorra essa modificação. Nessa etapa é essencial que o paciente entenda que o peso não é problema, mas, sim, as crenças que mantém os sintomas do transtorno (RANGÉ et al, 2011; OLIVEIRA; DEIRO, 2013; WILLHELM; FORTES; PERGHER, 2015).

As crenças centrais desses pacientes envolvem a hipervalorização acerca do peso, forma corporal, alimentação e valor pessoal. As crenças associam a magreza a competência, superioridade e sucesso. Em consequência a essa hipervalorização do peso e forma corporal há uma desconsideração de outras áreas da vida, como por exemplo, a social. Técnicas como a criação de um gráfico no "formato de pizza" em que são avaliados os graus de importância e investimento, que o paciente atribui a cada aspecto da sua vida podem ser utilizadas para que identifique e avalie a supervalorização dos aspectos relacionados ao peso, formato corporal, alimentação e capacidade de controle. O papel do terapeuta deve ser de modo colaborativo a fim de ajudar o paciente a identificar essa disfuncionalidade e investir em outros aspectos de sua vida (DUCHESNE; ALMEIDA, 2002; RANGÉ et al., 2011).

Indivíduos com TA tendem a serem muito críticos e perfeccionistas em relação ao próprio corpo e para isso é necessário um trabalho voltado à imagem corporal. $\mathrm{O}$ primeiro passo é demonstrar que o paciente se percebe de forma distorcida, para isso o terapeuta pode pedir ao paciente que desenhe como se percebe e em seguida o terapeuta contorne a silhueta do paciente, a fim de que perceba o tamanho real de seu corpo. Alguns pacientes evitam a exposição ao espelho e outros verificam de modo compulsivo e acentuam as imperfeições do corpo sob sua ótica. Para isso é importante que o terapeuta possa ajudar o paciente a identificar aspectos positivos, e para os pacientes com dificuldades de exposição, utilizar de técnicas de exposição gradual e guiada. De modo geral, o objetivo dessas técnicas é aumentar a autoaceitaçãoe ensinar o paciente a lidar com as eventuais "imperfeições", além disso, fazer com que o paciente perceba os diferentes biotipos e formatos de corpo (DUCHESNE; ALMEIDA, 2002; RANGÉ et al, 2011).

OTreinamento em Resolução de Problemas (TRP) e o desenvolvimento de estratégias para a regulação do humor são intervenções usadas para reduzir e regular estados intensos de humor em pacientes que apresentam episódios de compulsão alimentar e/ou usam de métodos compensatórios (RANGÉ et al, 2011).

No que tange a autoestima, a terapia cognitivocomportamental propõe desenvolver padrões realistas de autoavaliação com ênfase nos sucessos e qualidades, fazendo com que a autoestima fique apoiada em outros atributos além da aparência (DUCHESNE; ALMEIDA, 2002; RANGÉ et al, 2011).

Além desses aspectos, é relevante devolver as habilidades sociais que são frequentemente deficitárias em indivíduos com TA. Uma vez que são comuns crenças associadas à baixa competência para se comportar socialmente e à alta probabilidade de rejeição interpessoal (RANGÉ et al, 2011; OLIVEIRA; DEIRO, 2013; WILLHELM; FORTES; PERGHER, 2015).

-Estágio 4: Nesse estágio são realizadas a manutenção das conquistas anteriores e prevenção de recaídas através da antecipação, que consiste em identificar e planejar possíveis dificuldades e estratégias de enfrentamento a essas situações. Esse trabalho é realizado que modo colaborativo entre paciente e terapeuta (DUCHESNE; ALMEIDA, 2002). Nesse último estágio as sessões são realizadas em um intervalo de duas semanas (RANGÉ et al., 2011).

Os quatro estágios descritos acima apresentam características que integram técnicas que abordam fatores cognitivos, emocionais, comportamentais e interpessoais (RANGÉ et al., 2011).

\section{Considerações Finais}

A anorexia nervosa e a bulimia nervosa são transtornos alimentares que necessitam de um tratamento que contemple as múltiplas características etiológicas do transtorno. Com isso, o grande desafio para o tratamento adequado é justamente equilibrar as abordagens a serem utilizadas, com o uso de fármacos, com o ajustamento nutricional e a abordagem psicológica com técnicas cognitivo-comportamental, visto que as pesquisas demonstraram que a TCC tem maior popularidade e validade entre as demais abordagens (OLIVEIRA; DEIRO, 2013). Por meio de um tratamento multiprofissional atrelado ao apoio familiar é possível atingir bom prognóstico para os transtornos alimentares.

A TCC tem se mostrado eficaz para o tratamento da $\mathrm{AN}$, principalmente, em relação aoaumento da adesão ao tratamento nutricional e clínico;abordagem do distúrbio em relação à imagem corporal e a autoestima; modificações do sistema de crenças e pensamentos disfuncionais relacionados ao peso e alimentos;redução de recaídas e desenvolvimento das habilidades sociais que se encontram deficitárias (DUCHESNE; ALMEIDA, 2002). 
Enquanto para o tratamento da BN a TCC tem se mostrado relevante na remissão dos ciclos de compulsão alimentar-purgação; eliminação do uso de métodos compensatórios; na melhoria da autoestima e no aspecto social; e na modificação do sistema de crenças e dos pensamentos associados à alimentação e formato corporal(DUCHESNE; ALMEIDA, 2002; OLIVEIRA; DEIRO, 2013).

Devido à alta incidência dos transtornos alimentares, principalmente em mulheres adolescentes, é necessário que haja maior atenção e conhecimento por parte dos profissionais de saúde aos sintomas, curso e tratamento para um diagnóstico precoce, abordagem terapêutica e prognósticos adequados.

Tendo em vista que a utilização da TCC como tratamento para os TA ainda é um referencial relativamente recente no Brasil se faz necessário mais estudos sobre a temática, principalmente sobre o papel da família e os aspectos sociais no desenvolvimento, a manutenção e formação do transtorno; estudos sobre a epidemiologia e estratégias da TCC para o tratamento.

\section{Referências}

AMERICAN PSYCHIATRIC ASSOCIATION. Manual Diagnóstico e Estatítico de Transtornos Mentais: DSM- 5. $5^{\mathrm{a}}$ ed. Porto Alegre: Artmed, 2014

APPOLINÁRIO, J. C.; CLAUDINO, A. M. Transtornos alimentares. Revista Brasileira de Psiquiatria, São Paulo, v. 22, n. 2, p. 28-31, 2000

BECK, A. T. et al. Cognitivetherapyofdepression.The Guilford Press, New York, 1979.

BECK, J. S. Terapia Cognitivo- Comportamental: teoria e prática. 2 ed.Porto Alegre: Artmed, 2014

BUCARETCHI, H. A; CORDÁS, T. A. Distúrbios alimentares: anorexia e bulimia. In: QUAYLE, J.; LÚCIA, M. C. S., organizadores. Adoecer: as interações do doente com a sua doença. São Paulo: Atheneu; p. 49-60, 2003

CLAUDINO, A. M.; BORGES, M. B. F. Critérios diagnósticos para os transtornos alimentares: conceitos em evolução. Revista Brasileira de Psiquiatria, São Paulo, v. 24, n. 3, p. 7-12, 2002

DUCHESNE, M.; ALMEIDA, P. E. M. Terapia cognitivo-comportamental dos transtornos alimentares. Revista Brasileira de Psiquiatria, São Paulo, v. 24, n. 3, p. 49-53, 2002.

FLEITLICH, B. W. et al. Anorexia nervosa na adolescência. Jornal de Pediatria, Rio de Janeiro, v. 76, n. 3, p. 323-329. 2000

FORTES, L. S. et al. Modelo etiológico dos comportamentos de risco para os transtornos alimentares em adolescentes brasileiros do sexo feminino. Cadernos de Saúde Pública, Rio de Janeiro, v. 32, n. 4, p. 1-11, 2016.

GONÇALVES, M. Transtorno compulsivo alimentar periódico TCAP. Psychiatryonline Brasil, v. 17, n. 10, 2012.

HOEK, H.W.; VAN HOEKEN D. Review of the Prevalence and Incidence of Eating Disorders. International Journal of Eating Disorders, v. 34, p. 383396, 2003.

IDA, S.W.; SILVA, R.N. Transtornos alimentares: uma perspectiva social. Revista Mal- Estar e Subjetividade, v. 7, n. 2, p. 417-432, 2007.

MELIN, P.; ARAÚJO, A. M. Transtornos alimentares em homens: Um desafio diagnóstico. Revista Brasileira dePsiquiatria, v. 24, n. 3, p. 73-76, 2002.
MORGAN, C. M.; VECCHIATTI, I. R.; NEGRÃO, A. B. Etiologia dos transtornos alimentares: aspectos biológicos, psicológicos e sócio-culturais. Revista Brasileira de Psiquiatria, São Paulo, v. 24, n. 3, p. 18-23, 2002.

NationalInstitute for ClinicalExcellence(NICE). EatingDisorders: Core interventions in thetreatmentand management of anorexia, bulimia andrelatedeatingdisorders. ClinicalGuidelines, London, 2004

NUNES, M. A. et al. Transtornos alimentares e obesidade. 2. ed. Porto Alegre: Artmed, 2008.

OLIVEIRA, L. L.; DEIRO, C. P. Terapia Cognitivo-Comportamental para Transtornos Alimentares: A Visão de Psicoterapeutas sobre o Tratamento. Revista Brasileira de Terapia Comportamental e Cognitiva, São Paulo, v. 15, n. 1, p.36-49, 2013.

Organização Mundial da Saúde. CID-11 Classificação Estatística Internacional de Doenças e Problemas Relacionados à Saúde. São Paulo: Universidade de São Paulo, 2018.

PIZON, V.; NOGUEIRA, F.C. Epidemiologia, curso e evolução dos transtornos alimentares. Revista de Psiquiatria Clínica, São Paulo, v.31, n.4, p.158-160, 2004

PEDRINOLA, F. Nutrição e transtornos alimentares na adolescência. Pediatria Moderna, v 48, n. 7, p. 290-295, 2012

RANGÉ, B.et al. Psicoterapias Cognitivo-comportamentais: um diálogo com a psiquiatria. 2 ed. Porto Alegre: Artmed, 2011.

SALZANO, F. T.; CORDÁS, T. A. Tratamento farmacológico de transtornos alimentares. Revista de Psiquiatria Clínica, São Paulo, v. 31, n. 4, p. 188 194, 2004

SILVA, K. P. et al. Transtornos alimentares: considerações clínicas e desafios do tratamento. Infarma, Fortaleza, v. 19, n. 11/12, p. 9-12, 2007.

VILELA, J. E. M. et al. Transtornos alimentares em escolares. Jornal de Pediatria, Rio de Janeiro, v. 80, n. 1, p. 49-54, 2004

WILLHELM, A., FORTES, P., PERGHER, G. Perspectivas atuais da terapia cognitivo comportamental no tratamento dos transtornos alimentares: uma revisão sistemática. Revista Brasileira de Terapia Comportamental e Cognitiva, São Paulo, v.17, n. 2, p. 52-65, 2015

WRIGHT, J. H.; BASCO, M. R.; THASE, M. E. Aprendendo a terapia cognitivo-comportamental: um guia ilustrado. Porto Alegre: Artmed, 2008. 


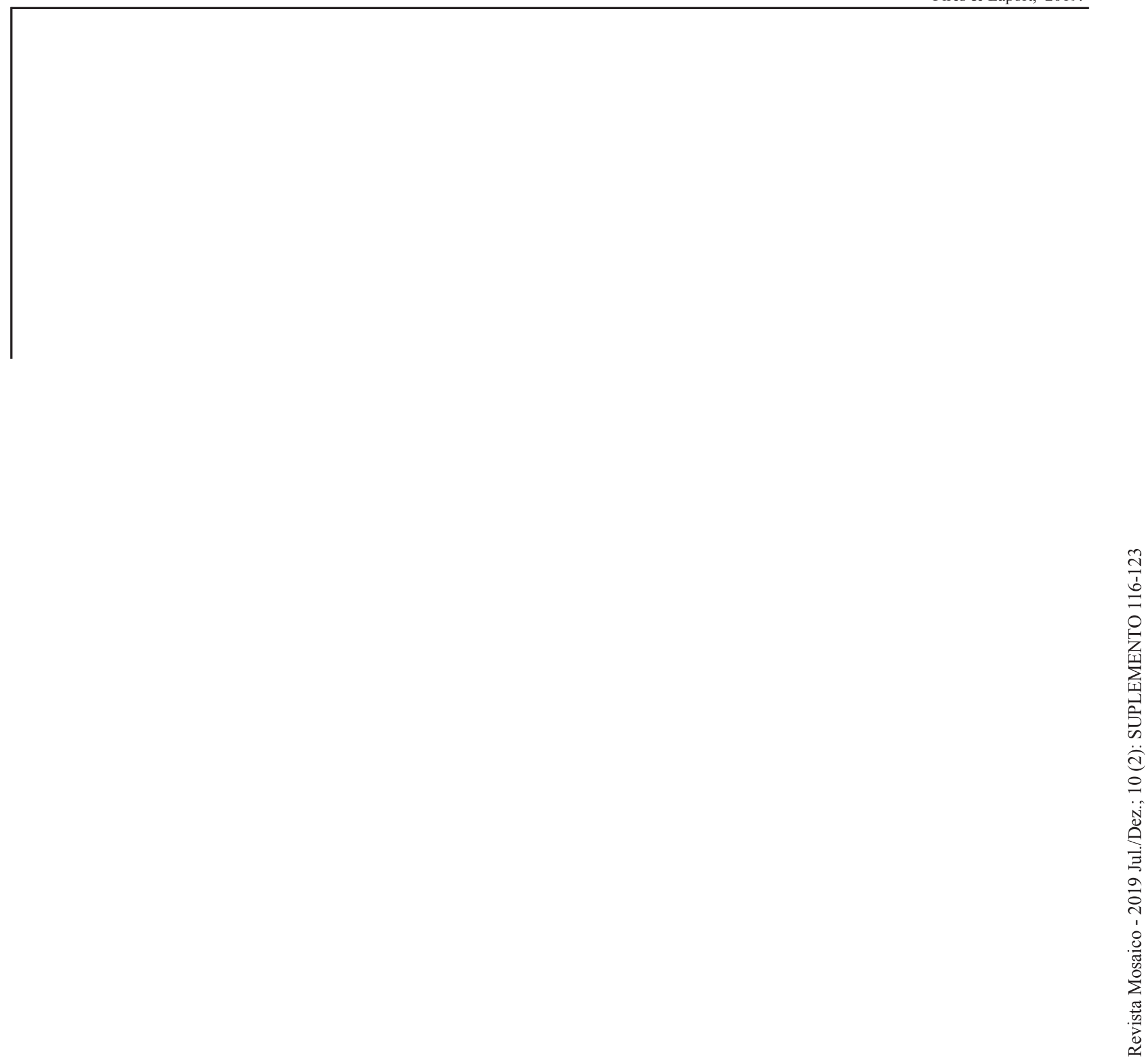

